TITLE:

\title{
Molecular dynamics study of laccase immobilized on self- assembled monolayer-modified Au
}

\section{$\operatorname{AUTHOR}(S)$ :}

Miyazawa, Naoki; Tanaka, Masahiro; Hakamada, Masataka; Mabuchi, Mamoru

\section{CITATION:}

Miyazawa, Naoki ...[et al]. Molecular dynamics study of laccase immobilized on selfassembled monolayer-modified Au. Journal of Materials Science 2017, 52(21): 1284812853

\section{ISSUE DATE:}

2017-11

URL:

http://hdl.handle.net/2433/237663

\section{RIGHT:}

This is a post-peer-review, pre-copyedit version of an article published in 'Journal of Materials Science'. The final authenticated version is available online at: https://doi.org/10.1007/s10853-017-1392-z/ The full-text file will be made open to the public on 17 July 2018 in accordance with publisher's 'Terms and Conditions for Self-Archiving'.; この論文 は出版社版でありません。引用の際には出版社版をご確認ご利用ください。; This is not the published version. Please cite only the published version. 


\section{Molecular Dynamics Study of Laccase Immobilized on Self-Assembled Monolayer Modified Au}

Naoki Miyazawa*, Masahiro Tanaka ${ }^{1}$, Masataka Hakamada, Mamoru Mabuchi

Graduate School of Energy Science, Kyoto University,

Yoshidahonmachi, Sakyo, Kyoto 606-8501, Japan

E-mail addresses:

miyazawa.naoki.37c@st.kyoto-u.ac.jp (N. Miyazawa)

marby20.6@gmail.com (M. Tanaka)

hakamada.masataka.3x@kyoto-u.ac.jp (M. Hakamada)

mabuchi@energy.kyoto-u.ac.jp (M. Mabuchi)

*Corresponding author. Tel.: +81 75753 5404; Fax: +81 757535428

E-mail address: miyazawa.naoki.37c@st.kyoto-u.ac.jp

Postal address: Graduate School of Energy Science, Kyoto University, Yoshidahonmachi,

Sakyo, Kyoto 606-8501, Japan 
${ }^{1}$ Present address: Sekisui Chemical Corporation, Toranomon, Minato-ku, Tokyo 1058405, Japan

\section{Abstract}

Molecular dynamics simulations of laccase immobilized by 4-aminothiophenol (4-ATP) were performed to understand the origin of the enhanced catalytic activity at $350 \mathrm{~K}$ of laccase immobilized by self-assembled monolayers (SAMs). The simulation showed that laccase was stabilized by bonding with 4-ATP. In addition, docking simulation of 2,6-dimethoxyphenol (DMP) to laccase revealed that the hydrophobic interaction energy was increased by bonding with 4-ATP. The variations of docking site size was minor considering the dimensions of DMP molecule. Therefore, it is suggested that the enhanced catalytic activity of laccase with 4-ATP is attributed to the high hydrophobic interaction energy between laccase and DMP.

Keywords: Laccase; Enzyme immobilization; Docking simulations; Molecular dynamics simulations 


\section{Introduction}

Free-standing nanoporous Au with large surface area can provide a platform for immobilization of self-assembled monolayers (SAMs) [1-3]. Au strongly bonds to the thiol group of SAM because of covalent bonding between an Au atom and a S atom in the thiol group of SAM [4]. Hakamada et al. [1] showed that the Au-S bond is strengthened by defects at the surfaces of nanoporous Au. Recently, it was found that laccase immobilized on SAM-modified nanoporous Au, where a 4-aminothiophenol (4ATP) was used as the SAM, exhibited enhanced catalytic activity in the degradation of 2,6-dimethoxyphenol (DMP) at a high temperature of $350 \mathrm{~K}$, compared with nonimmobilized laccase [5].

Laccase is a blue multicopper oxidase that contains four copper ions distributed in three sites, which are classified according to their spectroscopic properties, as type 1 $\mathrm{Cu}$ for the blue copper center, type $2 \mathrm{Cu}$ for the normal copper center, and type $3 \mathrm{Cu}$ for the coupled binuclear copper center. Type 1 (T1) Cu is the primary acceptor of electrons from a reducing substrate. Laccase is a useful enzyme that is capable of oxidizing a large number of organic and inorganic substrates [6], and it has received significant attention regarding its potential applications in the fields of biosensors and biofuel cells [7-9]. High stabilization of laccase at high temperature is important for such applications. Miyazaki 
[10] found that a hyperthermophilic laccase was produced by cloning the gene. Immobilization of laccase by a SAM is also one of methods for processing thermophilic laccase, as mentioned above. The immobilization of laccase by a SAM is promising for commercial applications because of its low cost. However, the origin of the enhanced catalytic activity at high temperature of laccase immobilized by a SAM is not understood at all.

In the present work, molecular dynamics (MD) simulations and docking simulations of laccase immobilized by 4-ATP were performed to understand the origin of the enhanced catalytic activity at high temperature. First, the bonding energy of laccase with 4-ATP was investigated at 300 and $350 \mathrm{~K}$. Then, the docking of DMP to laccase was simulated.

\section{Methods}

MD simulations of the bonding of laccase with and without 4-ATP were carried out using

Discovery Studio 4.0, using the CHARMM force field [11]. The crystal structure of laccase (PDB ID: 1V10 [12]) was obtained from the Protein Data Bank [13]. Laccase was immersed in a spherical water solvation where the number of water molecules was about 6,000 and the diameter of the sphere was $40 \mathrm{~nm}$. The time step of the MD simulation was 
$1 \mathrm{fs}$. The system was first energy-minimized using the steepest decent algorithm (200,000 steps) followed by the conjugate gradient method (200,000 steps). The system was gradually heated from 50 to 300 and $350 \mathrm{~K}$ for 2 ps, respectively. The system was equilibrated for 1 ns with constant number of particles, volume, and temperature (NVT) ensemble, where Berendsen's weak coupling sheme [14] was used to achieve constant temperature dynamics. Finally, 10 ns NVT simulations were performed. Laccase bonds with 4-ATP by formation of an amide bond between an amino group of 4-ATP and a carboxyl group of laccase $[15,16]$. In the present study, acidic amino acids positioned on the surface of laccase and C-terminal were bonded with 4-ATP. The amino acid in laccase that would most likely bond with 4-ATP was determined to be Glu91 because the lowest bonding energy was obtained for the bond between Glu91 and 4-ATP (Table 1). A schematic illustration of the amide bond between the amino group of 4-ATP and the carboxyl group of laccase is shown in Fig. 1. The bonding energy of laccase and 4-ATP is given by

$$
E_{\mathrm{b}}=E_{\text {laccase }+4 \mathrm{ATP}}-E_{\text {laccase }}-E_{4 \mathrm{ATP}}
$$

where $E_{\mathrm{b}}$ is the binding energy, and $E_{\text {laccase }+4 \mathrm{ATP}}$ Elaccase and $E_{4 \mathrm{ATP}}$, are the average internal energies of 10ns NVT simulations of laccase with 4-ATP, laccase, and 4-ATP, respectively. The bonding energies at 300 and $350 \mathrm{~K}$ were calculated by eq. (1). 
For docking simulations of DMP to laccase, AutoDock 4.2 software [17] was used to generate an ensemble of docked conformations for DMP and laccase. The Lamarckian genetic algorithm was used for all the molecular docking simulations and the following parameters were used; a random population of substrate conformations in up to 100 arbitrary orientations, a mutation rate of 0.02 , and a crossover rate of 0.8 . Simulations were carried out considering 2.5 million energy evaluations with a maximum of 27,000 generations. The docking site of DMP was determined to be T1 site because the substrate is reduced by $\mathrm{T} 1 \mathrm{Cu}$ and a previous study found that $\mathrm{T} 1$ site is the most energetically stable site for docking of a substrate to laccase [18]. The grid box composed of $40 \times 40 \times 40$ grid points with a spacing between each grid point of $0.375 \AA$ was centered a $\mathrm{T} 1 \mathrm{Cu}$. Laccase and $\mathrm{Cu}$ were rigid during docking simulations.

The hydrophobic energy was calculated using Poisson-Boltzmann solvent accessible surface area (MM-PBSA) method [19]. The 5 models were evenly extracted from the last 100 ps MD trajectories to calculate the hydrophobic interaction energy between laccase and DMP. The hydrophobic interaction energy can be calculated by

$$
E_{\mathrm{h}}=E_{\mathrm{h}, \text { laccase }+\mathrm{DMP}}-E_{\mathrm{h}, \text { laccase }}-E_{\mathrm{h}, \mathrm{DMP}}
$$

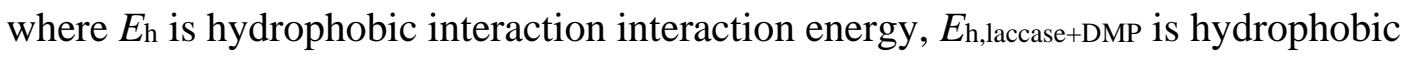
energy of laccase with DMP, $E_{\mathrm{h}, l a c c a s e}$ is hydrophobic energy of laccase and $E_{\mathrm{h}, \mathrm{DMP}}$ is 
hydrophobic energy of DMP. In the MM-PBSA calculation, a grid spacing of $0.5 \AA$ was employed and the relative dielectric constant was set to 80 at the exterior and 1.0 at the interior of DMP-laccase complex.

After docking simulations, the energy minimization, 1ns NVT equilibration and 10ns NVT simulations described above were performed again on the laccase with and without 4-ATP docked with DMP, respectively.

\section{Results and discussion}

\section{Stabilization of laccase with 4-ATP}

Fig. 2 shows variation of root-men square deviation (RMSD) as a function of calculation time for laccase with and without 4-ATP at 300 and 350K before docking with DMP, respectively. The RMSD value is given by,

$\operatorname{RMSD}=\sqrt{\frac{1}{n} \Sigma\left(x_{i}-X_{0}\right)^{2}}$

where $x_{0}$ is the initial atomic position, $x_{i}$ is the atomic position during the simulation, and $n$ is the number of atoms, respectively. The RMSD value was nearly constant after 8 ns and thus the structures of laccase were stable. The bonding energy of laccase with 4-ATP 
was $-47.1 \mathrm{kcal} / \mathrm{mol}$ at $300 \mathrm{~K}$ and was $-42.2 \mathrm{kcal} / \mathrm{mol}$ at $350 \mathrm{~K}$, respectively. Thus, laccase was stabilized by bonding with 4-ATP.

Fig. 3 shows the superposition of the part of laccase with and without 4-ATP, respectively. The structures of laccase did not overlap at $350 \mathrm{~K}$, while they overlapped at $300 \mathrm{~K}$, as shown by circles in Fig. 3. Thus, the structure of laccase changed more by bonding with 4-ATP at $350 \mathrm{~K}$ than at $300 \mathrm{~K}$. The conformation change leads to variation of the internal energy $[20,21]$. Therefore, it is suggested that the enhanced stabilization of laccase at $350 \mathrm{~K}$ by bonding with 4-ATP is because of the structural change of laccase.

\section{Docking of DMP to laccase}

Fig. 4 shows snapshots of laccase docked with DMP. DMP was docked near T1 Cu in all the cases. Some studies [22-24] suggested that structural change of laccase led to the low catalytic activity because the size of docking site became small. Hence, it is worthwhile to investigate a variation in size of $\mathrm{T} 1$ site. Figure 5 shows variations in $\mathrm{T} 1$ site size as a function of calculation time, where the T1 site size is defined as a diameter of a sphere which contains amino acids around T1 copper (His396, His457, Cys452 and Leu462). The average size of T1 site was $15.3 \AA$ for laccase without 4-ATP at $300 \mathrm{~K}, 15.6 \AA$ for 
laccase without 4-ATP at $350 \mathrm{~K}, 15.3 \AA$ for laccase with 4-ATP at $300 \mathrm{~K}$ and $14.8 \AA$ for laccase with 4-ATP at $350 \mathrm{~K}$, respectively. The dimensions of DMP molecule are $8.8 \AA$ along the long axis and $5.2 \AA$ along the short axis. Although the T1 site size for laccase with 4-ATP at $350 \mathrm{~K}$ was smaller than that for laccase without 4-ATP at $350 \mathrm{~K}$, a difference of the size was minor considering the dimensions of DMP molecule. Therefore, the enhanced catalytic activity of laccase with 4-ATP is unlikely to be related to the variation of T1 site size.

The hydrophobic interaction may play a vital role in an interaction between laccase and DMP because nonpolar amino acids such as Cys452 and Leu462 are located near T1 pocket [25]. Fig. 6 shows the hydrophobic interaction energy between laccase and DMP. It is noted that the hydrophobic interaction energy for laccase with 4-ATP was increased by a temperature increase from 300 to $350 \mathrm{~K}$, while it for laccase without 4ATP was decreased by the temperature increase. Therefore, it is suggested that the enhanced catalytic activity of laccase with 4-ATP is attributed to an enhancement in hydrophobic interaction.

A trade-off often occurs between the catalytic performance and the stability of laccase [26-28]. However, laccase immobilized by 4-ATP showed both high stability and high catalytic performance. Tokuriki et al. [28] noted that mutations that modulate 
enzymatic functions mostly destabilize, and mutations that stabilize seldom evolve enhanced enzymatic activity. The present work, however, suggests that structural changes caused by bonding with 4-ATP lead to the simultaneous high stability and high catalytic performance of laccase.

\section{Conclusion}

MD simulations and docking simulations of laccase immobilized on 4-ATP were performed to understand the origin of the enhanced catalytic activity at $350 \mathrm{~K}$ of laccase immobilized by a SAM. Laccase was stabilized by bonding with 4-ATP. The hydrophobic interaction energy between 2,6-dimethoxyphenol (DMP) and laccase was increased by bonding with 4-ATP. On the other hand, the variations of docking site size was minor considering the dimensions of DMP molecule. Therefore, enhanced catalytic activity of laccase with 4-ATP is attributed to the high hydrophobic interaction energy between laccase and DMP. 


\section{References}

[1] Hakamada M, Takahashi M, Furukawa T, Tajima K, Yoshimura K, Chino Y and Mabuchi M (2011) Electrochemical stability of self-assembled monolayers on nanoporous Au, Phys Chem Chem Phys. 13:12277-12284.

[2] Shulga O V, Jefferson K, Khan A R, D’Souza V T, Liu J, Demchenko A V and Stine K J (2007) Preparation and characterization of porous gold and its application as a platform for immobilization of acetylcholine esterase, Chem Mater. 19:3902-3911. [3] Qin H, Xu C, Huang X, Ding Y, Qu Y and Gao P (2009) Immobilization of laccase on nanoporous gold: comparative studies on the immobilization strategies and the particle size effect, J Phys Chem. C 113:2521-2525.

[4] Tielens F and Santos E (2010) AuS and SH bond formation/breaking during the formation of alkanethiol SAMs on Au (111): a theoretical study, J. Phys Chem. C 114:9444-9452.

[5] Hakamada M, Takahashi M and Mabuchi M (2012) Enhanced thermal stability of laccase immobilized on monolayer-modified nanoporous Au, Mater Lett. 66:4-6.

[6] Yaropolov A, Skorobogat'ko O V, Vartanov S S and Varfolomeyev S D (1994) Laccase properties, catalytic mechanism, and applicability, Appl Biochem Biotechnol. 49:257-280. 
[7] Gupta G, Rajendran V and Atanassov P (2003) Laccase biosensor on monolayermodified gold electrode, Electroanalysis 20:1577-1583.

[8] Mena M L, Carralero V, González-Cortés A, Yáñez-Sedeño P and Pingarrón J M (2005) Laccase biosensor based on N-succinimidyl-3-thiopropionate-functionalized gold electrodes, Electroanalysis 23:2147-2155.

[9] Deng L, Wang F, Chen H, Shang L, Wang L, Wang T and Shaojun D (2008) A biofuel cell with enhanced performance by multilayer biocatalyst immobilized on highly ordered macroporous electrode, Biosens Bioelectron. 24:329-333.

[10] Miyazaki K (2005) A hyperthermophilic laccase from Thermus thermophilus HB27, Extremophiles 9:415-425.

[11] MacJerell A D Jr, Bashford D and Bellott M et al (1998) All-atom empirical potential for molecular modeling and dynamics studies of proteins, J Phys Chem. B 102:3586-3616.

[12] Garavaglia S, Cambria M T, Miglio M, Ragusa S, Lacobazzi V, Palmieri F, D’Ambrosio C, Scaloni A and Rizzi M (2004) The structure of Rigidoporus lignosus Laccase containing a full complement of copper ions, reveals an asymmetrical arrangement for the T3 copper pair, J Mol Biol. 342:1519-1531. 
[13] Berman H M, Westbrook J, Feng Z and Gilliland G et al (2000) The protein data bank, Nucleic Acids Res. 28:235-242.

[14] Berendsen H J C, Postma J P M, DiNola A and Haak J R (1984) Molecular dynamics with coupling to an external bath, J. Chem. Phys. 81:3684-3690.

[15] Dominguez C V, Pita M, De Lacey A L, Shleev S and Cuesta A (2012) Combined ATR-SEIRAS and EC-STM study of the immobilization of laccase on chemically modified Au electrodes, J. Phys. Chem. C 116:16532-16540.

[16] Gupta G, Rajendran V and Atanassov P (2004) Bioelectrocatalysis of oxygen reduction reaction by laccase on gold electrodes, Electroanalysis 16:1182-1185.

[17] Morris G M, Huey R, Lindstrom W, Sanner M F, Belew R K, Goodsell D S and Oison A J (2009) AutoDock4 and AutodockTools4: automated docking with selective receptor flexibility, J. Compt. Chem. 30:1639-1662.

[18] Martinez-Sotres C, Rutiaga-Quinones J G, Herrera-Bucio R, Gallo G and LopezAlbarran P (2015) Wood. Sci. Technol. 49:857-868.

[19] Srinivasan J, Cheatham T E, Cieplak P, Kollman P A and Case D A (1998)

Continuum solvent studies of the stability of DNA, RNA, and phosphoramidate-DNA helices, J Am. Chem. Soc., 120:9401-9409. 
[20] Trevino S R, Schaefer S, Scholtz J M and Pace C N (2007) Increasing protein conformational stability by optimizing beta-turn sequence, J. Mol. Biol. 373:211-218.

[21] Fu H, Grimsley G R, Razvi A, Scholtz J M and Pace C N (2009) Increasing protein stability by improving beta-turns, Proteins 77:491-498.

[22] Wang F, Guo C, Liu H -Z and Liu C -Z (2008) Immobilization of Pycnoporous sanguineus laccase by metal affinity adsorption on magnetic chelator particles, J. Chem. Technol. Biotech. 83:97-104.

[23] Bayramoglu G, Yilmaz M and Arica M Y (2010) Preparation and characterization of epoxy-functionalized magnetic chitosan beads: laccase immobilized for degradation of reactive dyes, Bioprocess Biosyst. Eng. 33:439-448.

[24] Fernandes R A, Daniel-da-Silva A L, Tavares A P M and Xavier A M R B (2017) EDTA-Cu(II) chelating magnetic nanoparticles as a support for laccase immobilization, Chem. Eng. Sci. 158:599-605.

[25] Garavaglia S, Cambria M T and Miqilio M et al. (2004) The structure of Rigidporous Lignisus Laccase containing a full complement of copper ions, reveals an asymmetrical arrangement for the T3 copper pair, J. Mol. Biol. 342:1519-1531. 
[26] Autore F, Del Vecchio C, Fraternali F, Giardina P, Sannia G and Faraco V (2009)

Molecular determinants of peculiar properties of a Pleurotus ostreatus laccase: analysis

by site-directed mutagenesis, Enzyme Micro Tech. 45:507-513.

[27] Vieille C and Zeikus G J (2001) Hyperthermophilic Enzymes: Sources, Uses, and

Molecular Mechanisms for Thermostability, Mirobial Mol Bio Rev. 65:1-43.

[28] Tokuriki N, Stricher F, Serrano L and Tawfik D S (2008) How protein stability and new functions trade off. PLoS Comput. Biol., 4:e1000002. 
Table 1 The binding energies of 4-ATP with acidic amino acids positioned on the surface of laccase and C-terminal (Asn494), respectively. The lowest binding energy was obtained in Glu91.

\begin{tabular}{|c|c|c|c|c|c|c|}
\hline $\begin{array}{c}\text { Amino } \\
\text { Acid }\end{array}$ & Glu28 & Glu91 & Clu254 & Glu293 & Asp469 & Asn494 \\
\hline $\begin{array}{c}\text { Binding } \\
\text { Energy } \\
\text { (kcal/mol) }\end{array}$ & -10.8 & -47.2 & -41.4 & -19.8 & -32.3 & 23.1 \\
\hline
\end{tabular}




\section{Figure Captions}

Fig. 1 Illustration of the amide bond between the amino group of 4-ATP and a carboxyl group of laccase at $300 \mathrm{~K}$. The amino acid in laccase is Glu91.

Fig. 2 Variations in RMSD value as a function of calculation time for laccase with and without 4-ATP, (a) laccase without 4-ATP at $300 \mathrm{~K}$, (b) laccase without 4-ATP at $350 \mathrm{~K}$, (c) laccase with 4-ATP at $300 \mathrm{~K}$ and (d) laccase with 4-ATP at $350 \mathrm{~K}$. The RMSD value becomes nearly constant after 8ns.

Fig. 3 Snapshots of superposition of parts of laccase with and without 4-ATP after MD simulation at (a) $300 \mathrm{~K}$ and (b) $350 \mathrm{~K}$. The part of laccase with and without 4-ATP after the stabilization calculations are shown in red and green, respectively. The structure of laccase does not overlap at $350 \mathrm{~K}$, while they overlap at $300 \mathrm{~K}$, as shown by circles.

Fig. 4 Snapshots of conformation of laccase docked with DMP after MD simulation, (a) laccase without 4-ATP at $300 \mathrm{~K}$, (b) laccase without 4-ATP at $350 \mathrm{~K}$, (c) laccase with 4ATP at $300 \mathrm{~K}$ and (d) laccase with 4-ATP at $350 \mathrm{~K}$. DMP is docked at T1 pocket. 
Fig. 5 Variations in T1 site size as a function of calculation time. The T1 site size is defined as a diameter of a sphere which is drawn by approximating a receptor range containing His396, His457, Cys452 and Leu462.

Fig. 6 Hydrophobic interaction energy between laccase and DMP. Hydrophobic interaction energy decreases from 300 to $350 \mathrm{~K}$ for laccase without 4-ATP and increases for laccase with 4-ATP. 


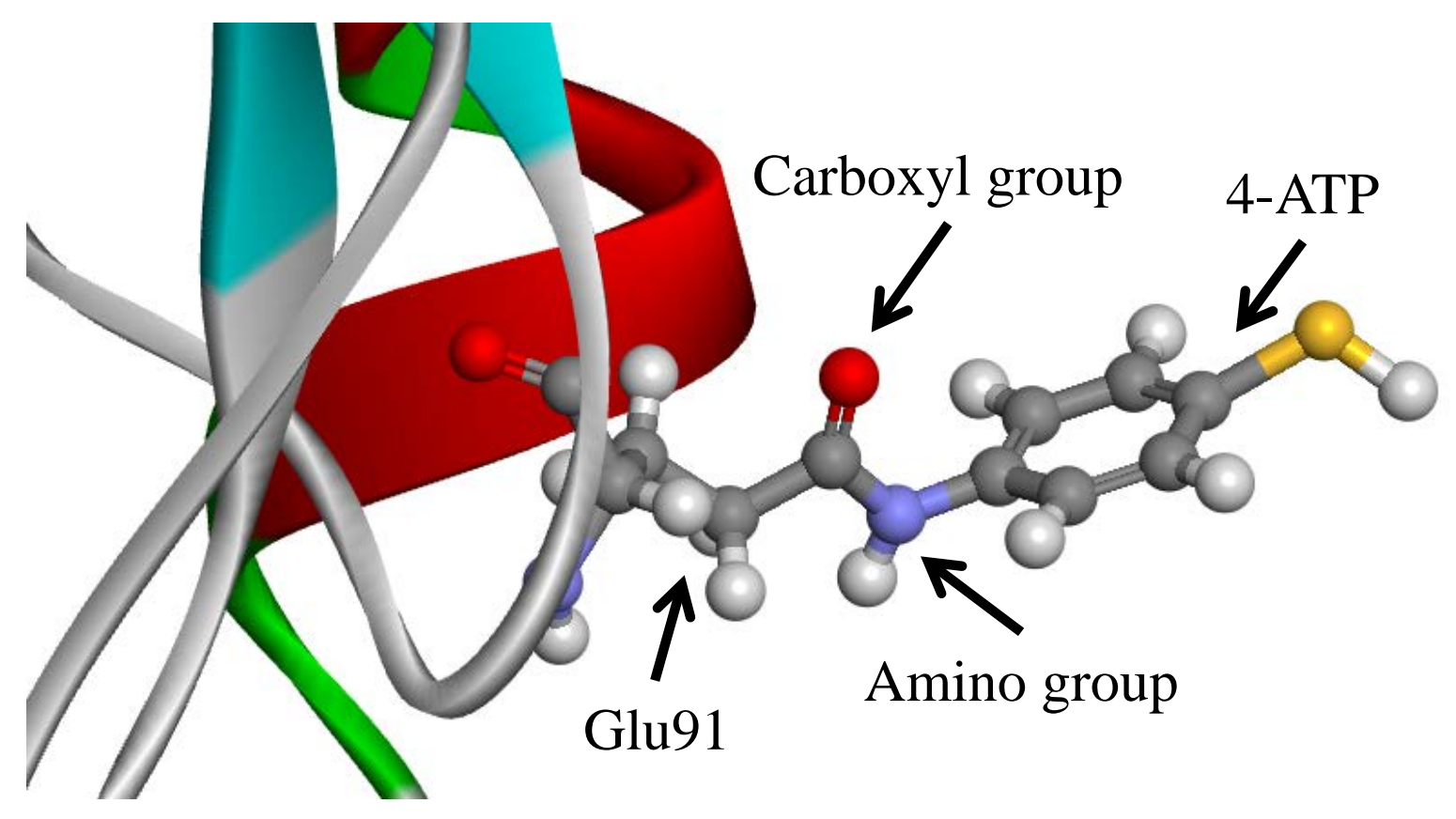

Figure 1 


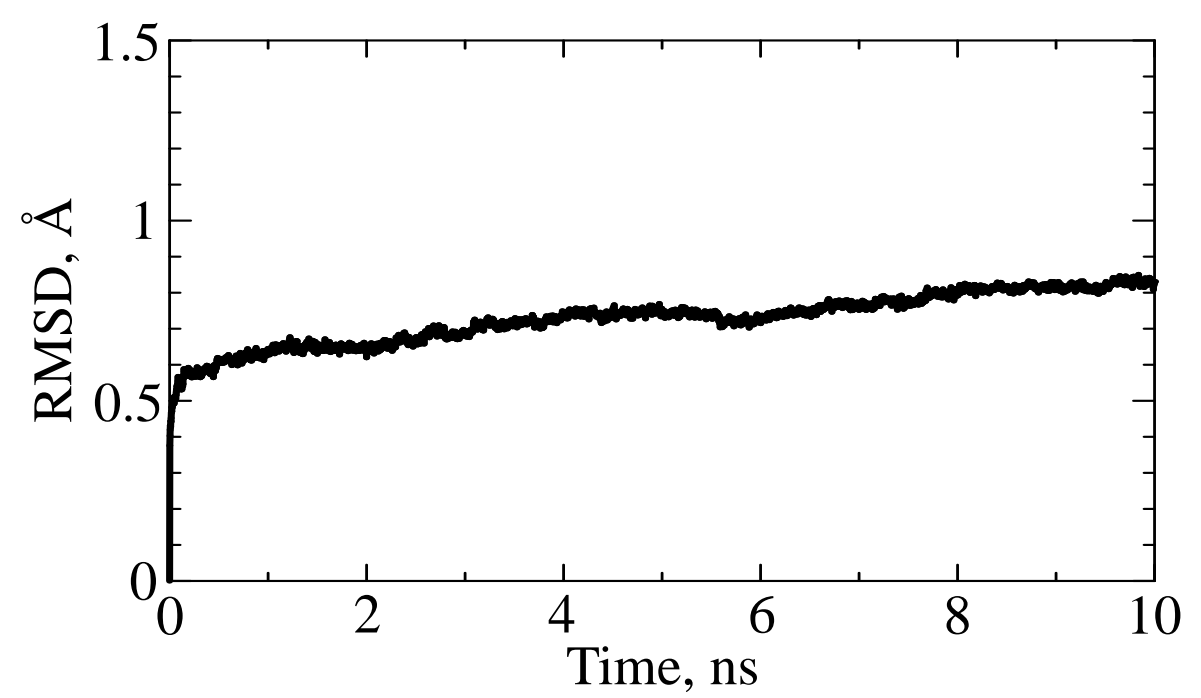

(a)

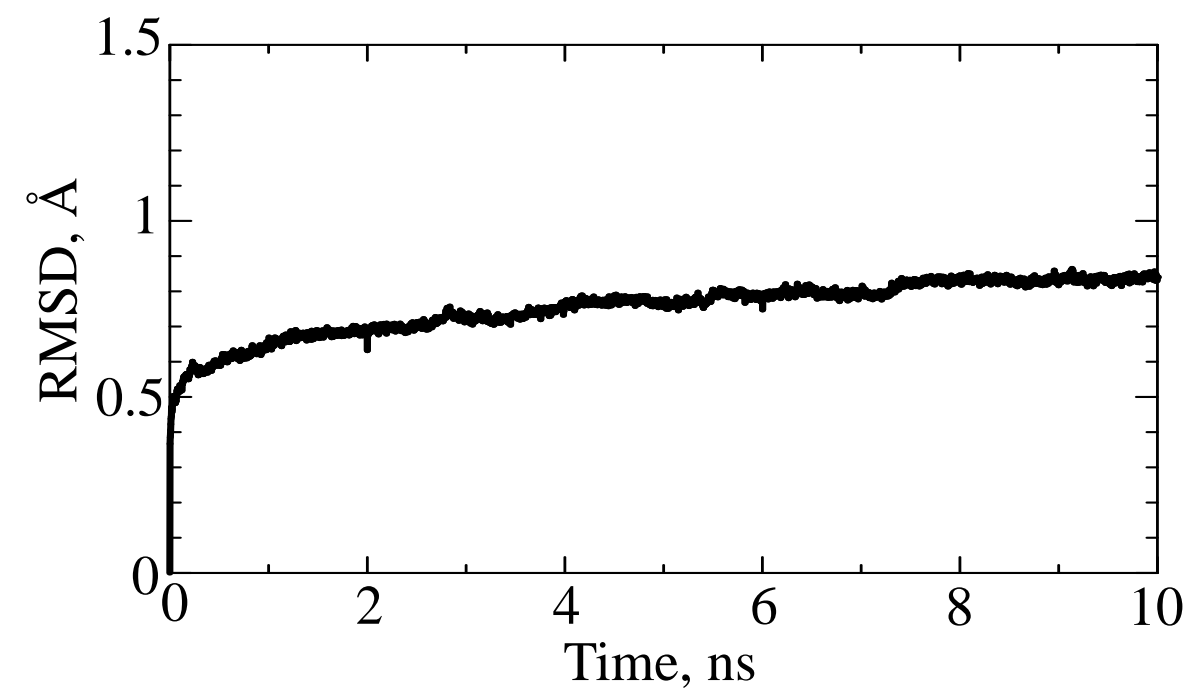

(c)

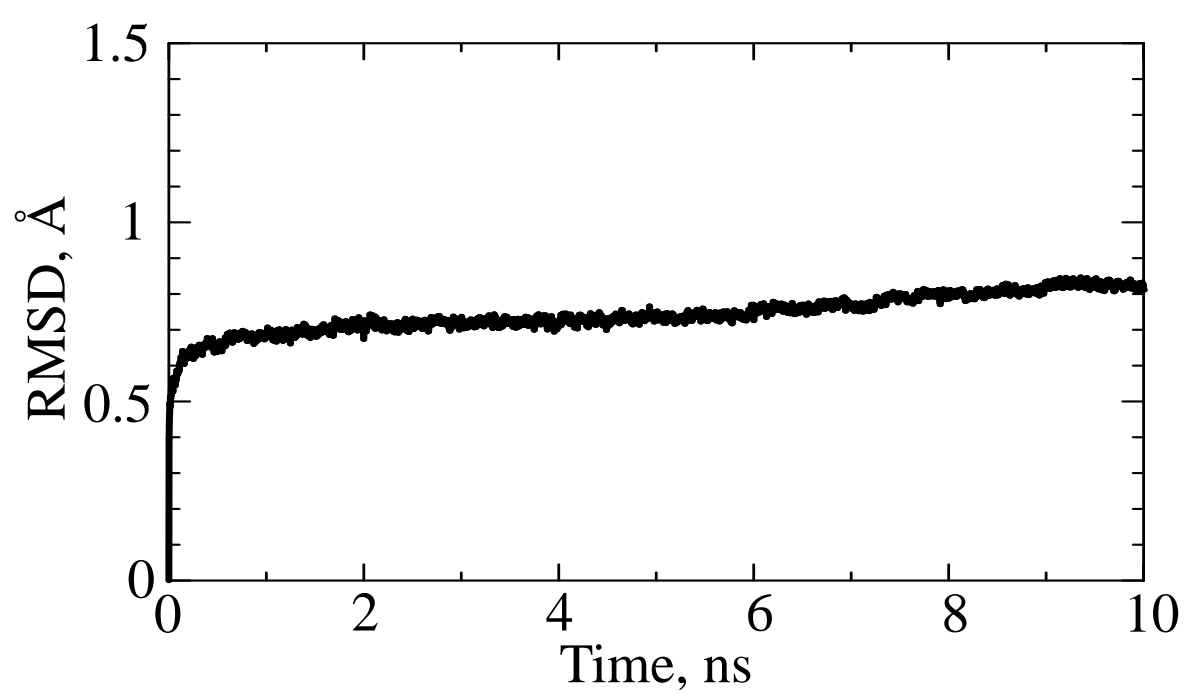

(b)

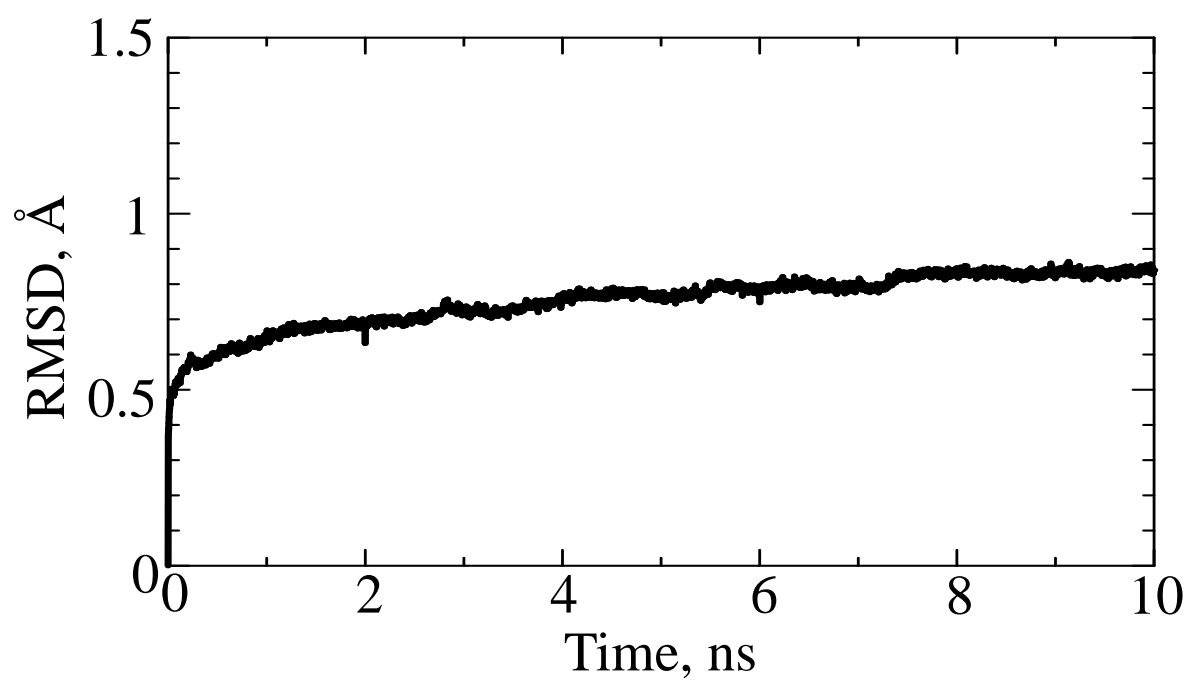

(d)

Figure 2 


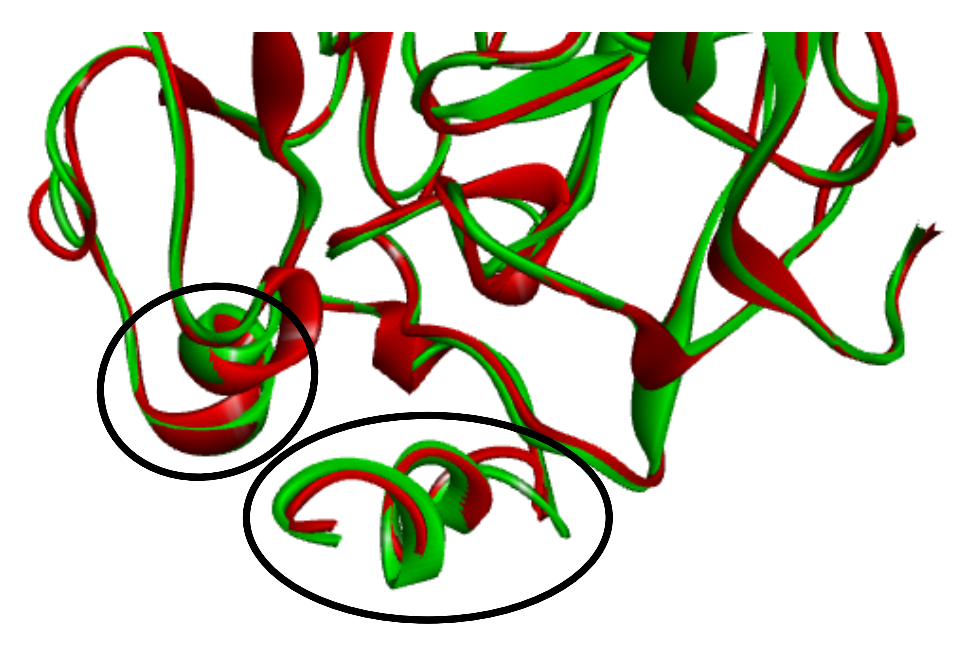

(a)

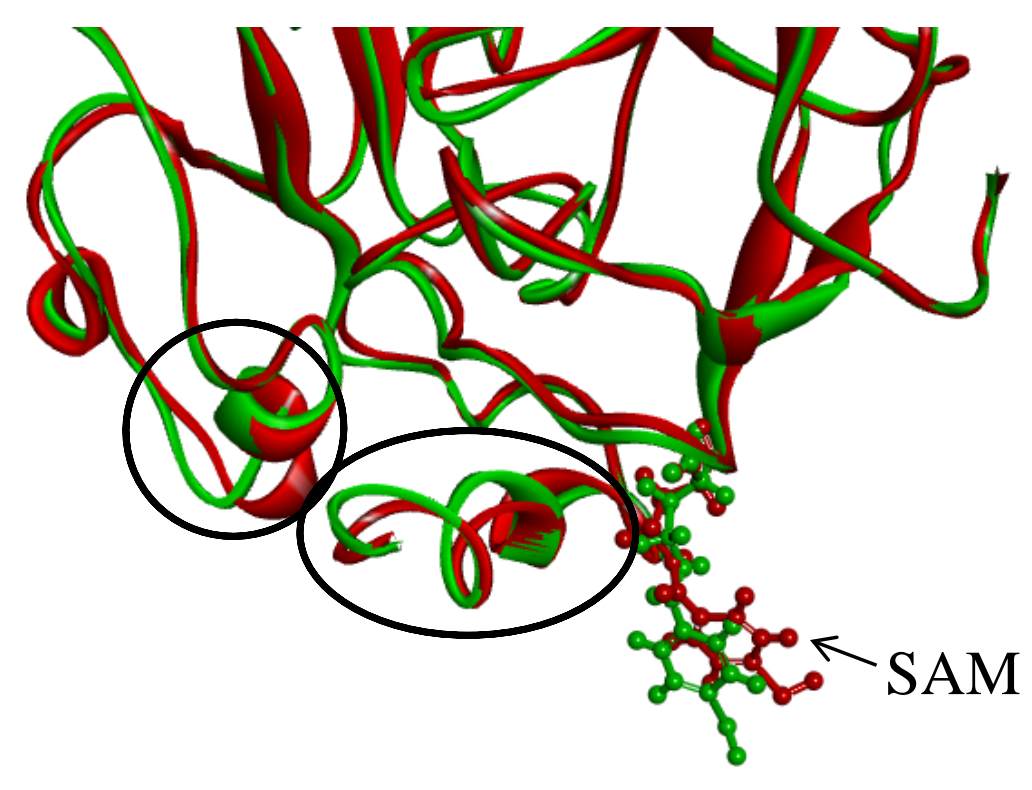

(b)

Figure 3 


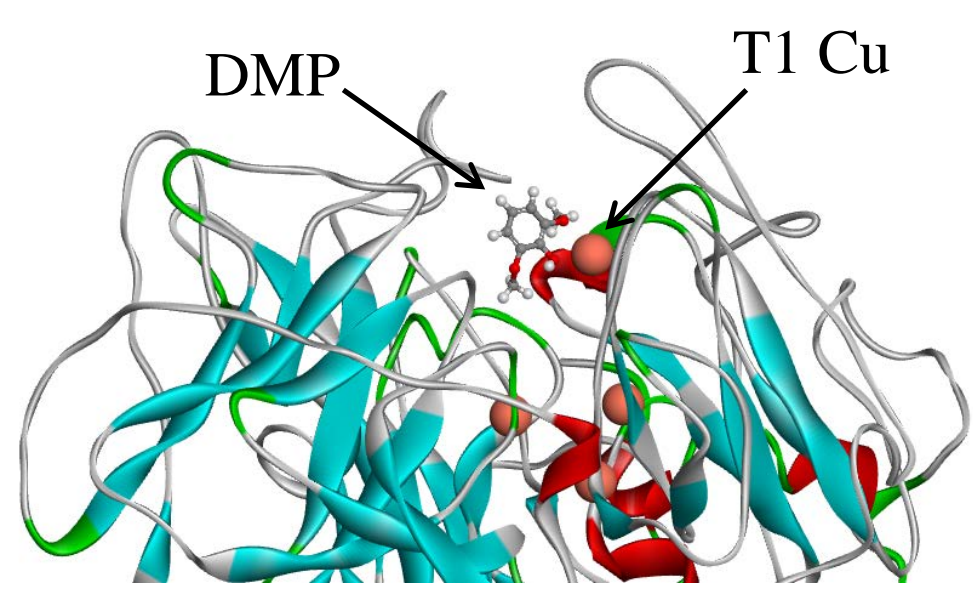

(a)

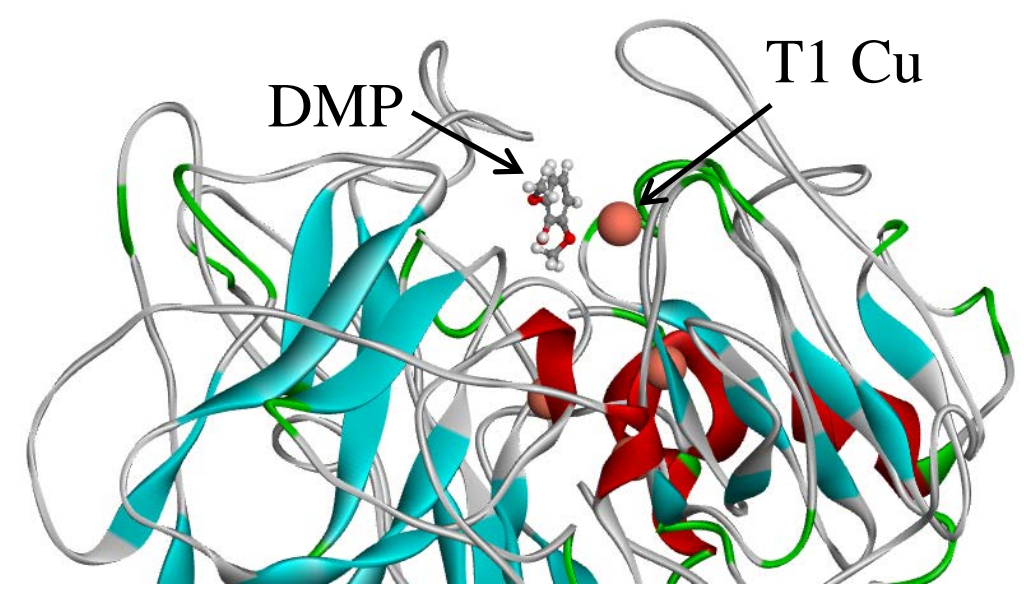

(c)

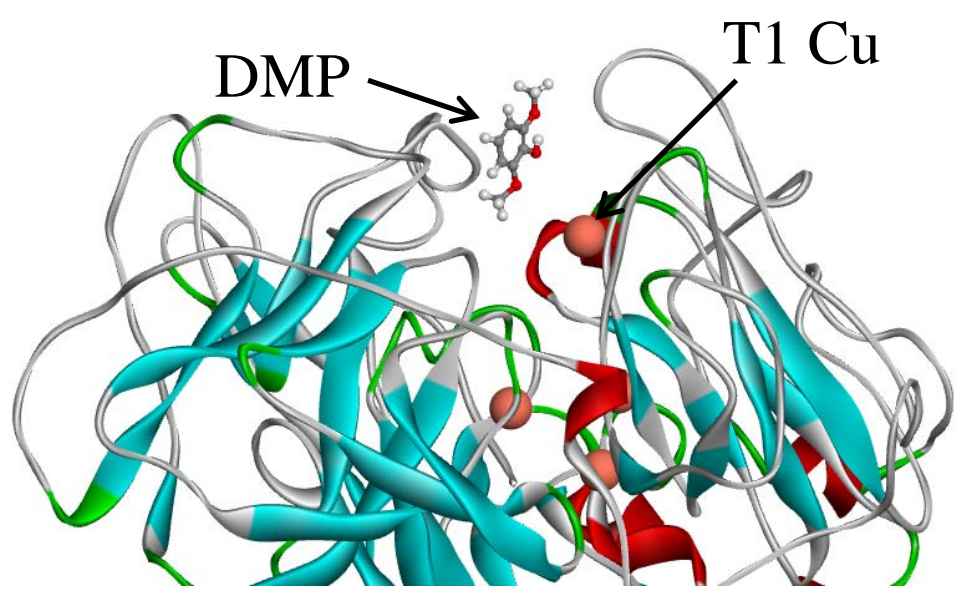

(b)

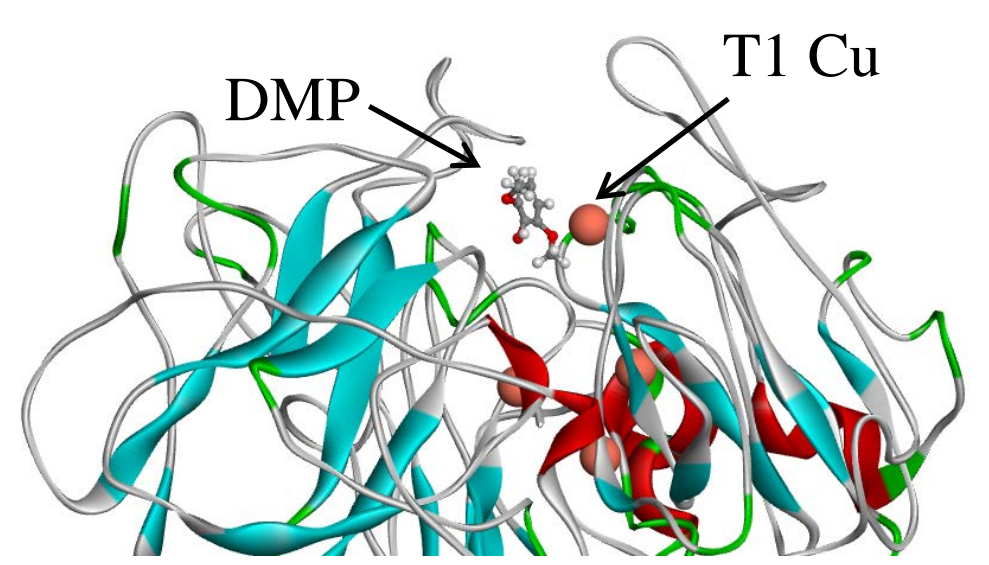

(d)

Figure 4 


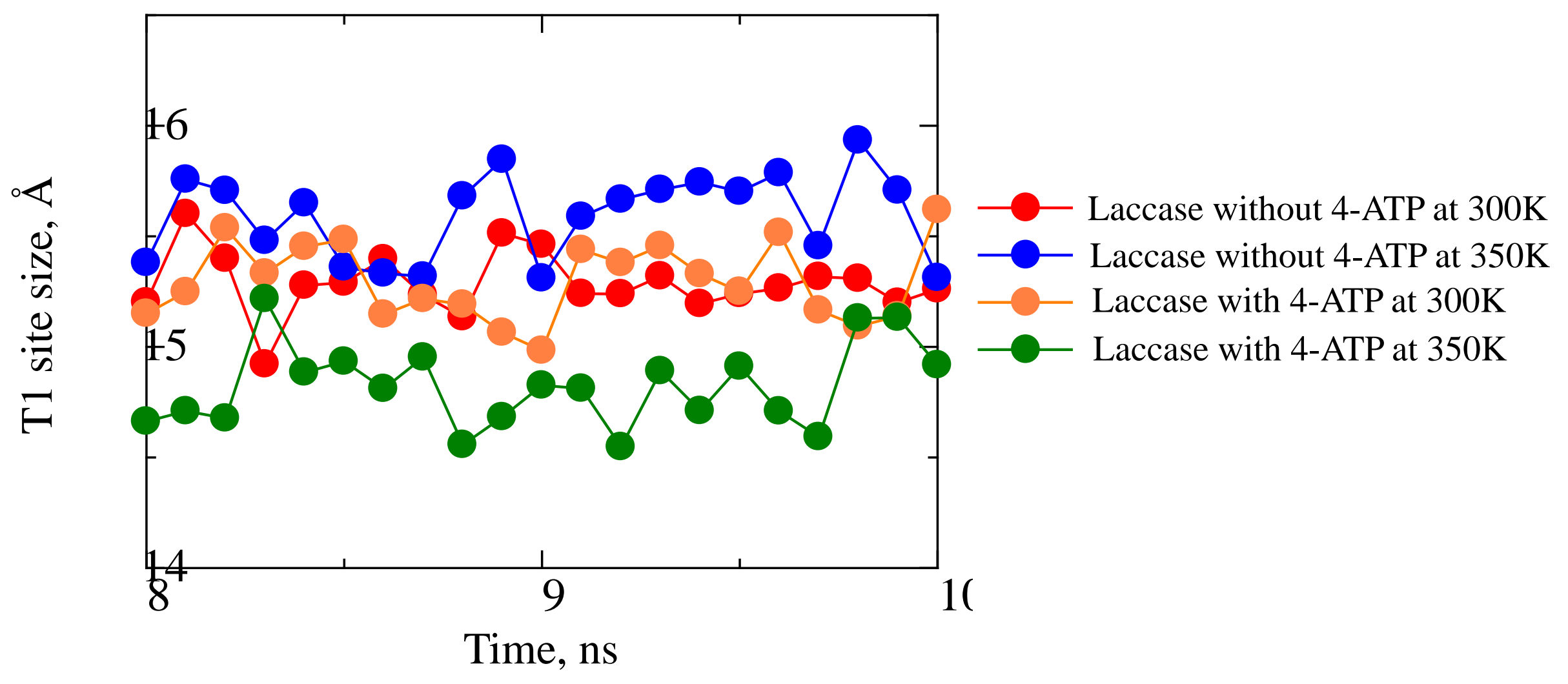

Figure 5 


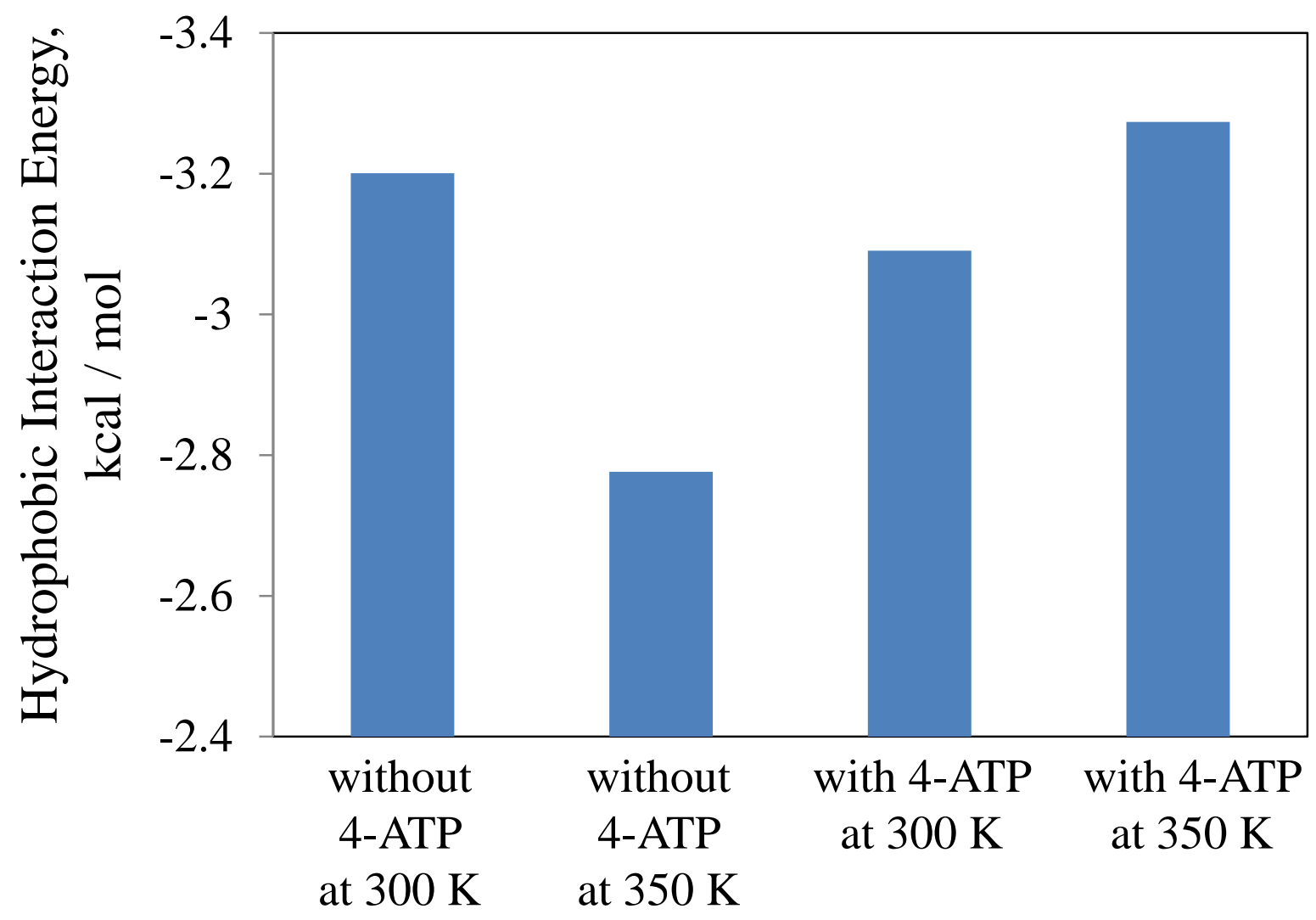

Figure 6 